\title{
NONRESONANT INTERACTION OF ULTRASHORT ELECTROMAGNETIC PULSES WITH MULTILEVEL QUANTUM SYSTEMS
}

\author{
E.Belenov, V.Isakov and A.Nazarkin \\ Lebedev Physics Institute, Russian Academy of Sciences, Leninsky pr. 53, 117924 Moscow, \\ Russia
}

\begin{abstract}
Some features of the excitation of multilevel quantum systems under the action of electromagnetic pulses which are shorter than the inverse frequency of interlevel transitions are considered. It is shown that the interaction is characterized by a specific type of selectivity which is not connected with the resonant absorption of radiation. The simplest three-level model displays the inverse population of upper levels. The effect of an ultrashort laser pulse on a multilevel molecule was regarded as an instant reception of the oscillation velocity by the oscillator and this approach showed an effective excitation and dissosiation of the molecule. The estimations testify to the fact that these effects can be observed using modern femtosecond lasers.
\end{abstract}

\section{Introduction}

Progress in ultrashort pulse technique allows production of laser pulses of 5-10 femtosecond duration, i.e., of a few periods of electromagnetic wave [1]. The interaction of so short light pulses with matter must differ from the case of quasi-monochromatic resonant radiation because the pulse duration becomes shorter than the inverse frequancy of the transitions between vibrational levels of molecules $\omega_{\mathrm{ij}}^{-1}$ or optical phonons in crystals, and under such conditions the result of interaction is practically independent of the real structure of matter. In particular, a Raman-active medium can be effectively excited by a single femtosecond pulse because its wide spectrum initially contains Stokes and anti-Stokes components of the field $[2,3]$.

We shall discuss here two effects which may occur if the light pulse is shorter than the time of vibrational transitions between molecule levels, i.e. under the condition $\tau_{\mathbf{p}} \omega_{\mathrm{ij}} \ll 1$. The first effect displays a specific selectivity of vibrational excitation of molecules by ultrashort light pulses; which is not connected with resonant properties of matter. The second effect is a possibility of effective excitation of high vibrational levels and even dissosiation of molecules in the field of an ultrashort electromagnetic pulse.

PRGCEOHO PAGE DARK NOT FKMED

PAGE $5 / 4$ INTENTONALLY BLANG 515 


\section{Selectivity of Molecule Excitation by Nonresonant Pulse Radiation}

The selectivity of molecule excitation is connected with the fact that the probability of transitions between molecule levels cannot be presented already as a function of slowly varying amplitude $\mathrm{E}(\mathrm{t})$ of the light field similar to the case of quasimonochromatic resonant radiation but depends on its real temporal structure $\mathcal{E}(t)$. The equations for ultrashort pulse propagation, in their turn, impose restrictions on such integral values as $\int_{-\infty}^{\tau} \mathcal{E}(t) d t$. As shown in Ref [4], the "area" of electromagnetic wave of limited aperture in a free space goes to zero, $\int_{-\infty}^{\infty} \mathcal{E}(t) d t=0$. It results from the Gauss' integral theorem in the case of electromagnetic wave propagation in a space without electric charges. If $\mu_{n m}$ is a dipole moment of the transition between levels $\mathrm{m}$ and $\mathrm{n}$, the amplitude of the transition $n \rightarrow m$ in the dipole approximation is of the form

$$
a_{m}^{(1)}(\infty)=-\frac{i}{\hbar} \int_{-\infty}^{\infty} \mu_{n m} \mathcal{E}(t) e^{i \omega_{n m} t} d t \stackrel{\tau_{p} \omega_{n m}<1}{\longrightarrow}-\frac{i \mu_{n m}}{\hbar} \int_{-\infty}^{\infty} \mathcal{E}(t) d t
$$

and tends to zero for ultrashort pulses.

It means that excitation of vibrational levels of molecules by femtosecond pulses is mainly due to two-quanta processes over distant virtual levels

$$
a_{m}^{(2)}(\infty)=-\frac{i}{2 \hbar} \int_{-\infty}^{\infty} r_{n m} E^{2}(t) e^{i \omega_{n m} t} d t
$$

where $r_{n m}=\frac{1}{2 \hbar} \sum_{k} \mu_{n k} \mu_{k m}\left(\frac{1}{\omega_{k n}-\omega}+\frac{1}{\omega_{k m}+\omega}\right)$ is a composite matrix element responsible for Raman effect at the transition $n \rightarrow m$, and the probability of excitation in the second order perturbation theory is proportional to the pulse energy $\int_{-\infty}^{\infty}|E(t)|^{2} d t \neq 0$. Thus, an ultrashort pulse provides "inverse" selectivity of molecule excitation - the transitions forbidden in the dipole approximation are stimulated much more effectively than the allowed ones.

As an illustration let us consider three-level quantum system, i.e. three lowest levels of the deformational vibrations of the $C \mathrm{O}_{2}$ molecule $\left(00^{\circ} 0,01^{\circ} 0\right.$ and $\left.02^{\circ} 0\right)$. The model assumes that the condition of the ultrashort interaction $\tau_{p} \omega_{i j} \ll 1$ holds for $i, j=1,2,3$ and the other levels are distant in the energy scale, i.e. $\left|\omega_{i k}-\omega\right| \gg \tau_{p}^{-1}, k \neq 1,2,3$. Levels (1) and (3) are of the same parity and the transition between them is forbidden in the dipole approximation.

The condition $\tau_{p} \omega_{i j} \ll 1$ implies of course that the interaction of an ultrashort pulse with matter is coherent $\left(\tau_{p} \ll T_{1}, T_{2}\right.$, pulse duration is shorter than times of longitudinal and transverse relaxcation) and can be described by equations for the probability amplitudes of the molecule to be at the level $\mathrm{j}(=1,2,3)$ :

$$
\begin{aligned}
i \hbar \frac{d a_{1}}{d t} & =-\mu_{12} \mathcal{E}(t) a_{2}-r_{13} \mathcal{E}^{2}(t) a_{3}, \\
i \hbar \frac{d a_{2}}{d t} & =-\mu_{21} \mathcal{E}(t) a_{1}-\mu_{23} \mathcal{E}(t) a_{3}, \\
i \hbar \frac{d a_{3}}{d t} & =-\mu_{32} \mathcal{E}(t) a_{2}-r_{31} \mathcal{E}^{2}(t) a_{1} .
\end{aligned}
$$

Let the system be at the ground level (1) at the initial instant of time, i.e. $a_{1}(t=-\infty)=1$ and $a_{2}(t=-\infty)=a_{3}(t=-\infty)=0$. Under the action of ultrashort pulse, the transitions onto 
level (2) occur due to the fast oscillating field $\mathcal{E}(\mathrm{t})$ which do not have a constant component, but transitions onto level (3) occur due to the interaction quadratic in field and $\overline{\mathcal{E}^{2}(t)} \neq 0$. We can assume that during the interaction the redistribution of population will be provided mainly by the transitions over level (3). Indeed, by integrating the second equation and saving here the amplitudes $\bar{a}_{1}$ and $\bar{a}_{3}$ whose time dependence is slow with respect to $\mathcal{E}(t)$, one obtains

$$
a_{2}(t) \simeq \frac{i}{\hbar}\left[\mu_{21} \bar{a}_{1}(t)+\mu_{23} \bar{a}_{3}(t)\right] \int_{-\infty}^{t} \mathcal{E}(t) d t .
$$

In other words, the population of level (2) is small $\left(a_{2} \sim \mu_{i j} \mathcal{E} / \hbar \omega\right)$ because of a nonresonant interaction of this level with the field of the ultrashort pulse. After substitution of $a_{2}(t)$ into the first and third equations one can obtain equations for slow components of the amplitudes $\bar{a}_{1}$ and $\bar{a}_{3}$, which do not contain parameters of dipole transitions $1 \rightarrow 2$ and $2 \rightarrow 3$ :

$$
\begin{aligned}
i \hbar \frac{d \bar{a}_{1}}{d t} & =-\frac{r_{13}}{2}|E(t)|^{2} \bar{a}_{3}, \\
i \hbar \frac{d \bar{a}_{3}}{d t} & =-\frac{r_{31}}{2}|E(t)|^{2} \bar{a}_{1} .
\end{aligned}
$$

It is interesting to note that the solution of these equations

$$
\begin{aligned}
\bar{a}_{1}(t) & =\cos \Psi(t), \\
\bar{a}_{3}(t) & =i \sin \Psi(t), \\
\Psi(t) & =\frac{r_{13}}{2 \hbar} \int_{-\infty}^{t}|E(t)|^{2} d t
\end{aligned}
$$

coincides formally with the solution for resonant coherent excitation of two-quanta transition, although in our case the interaction is nonresonant in principle.

The population of molecule levels after the action of ultrashort pulse is determined by the following expressions:

$$
\begin{aligned}
& n_{1}(\infty)=\cos ^{2}\left(\frac{r_{13}}{2 \hbar} \int_{-\infty}^{\infty}|E|^{2} d t\right), \\
& n_{2}(\infty)=\frac{1}{\hbar^{2}}\left[\mu_{12}^{2} n_{1}(\infty)+\mu_{23}^{2} n_{3}(\infty)\right]\left(\int_{-\infty}^{\infty} \mathcal{E}(t) d t\right)^{2}=0, \\
& n_{3}(\infty)=\sin ^{2}\left(\frac{r_{13}}{2 \hbar} \int_{-\infty}^{\infty}|E|^{2} d t\right) .
\end{aligned}
$$

These equations display an evident selectivity of the interaction of an ultrashort light pulse with the three-level quantum system which produces the population inversion on the transition $3 \rightarrow 2$. If the energy of an ultrashort pulse corresponds to the value of the rotation phase $\Psi_{\infty}=\frac{r_{13}}{2 \hbar} \int_{-\infty}^{\infty}|E|^{2} d t=\frac{\pi}{2}(2 n+1), n=0,1,2, \ldots$ the quantum system will be moved onto the upper level (3) and all the energy stored can be picked up, for example, by two sequential $\pi$-pulses which are resonant to transitions $3 \rightarrow 2$ and $2 \rightarrow 1$.

Let us make some estimations for the molecule $\mathrm{CO}_{2}$. It can be easily shown that if the initial population $n_{20}$ of the level (2) is not equal to zero, the inversion on the transition $3 \rightarrow 2$ appears if $\Psi_{\infty}>\left(n_{20} / n_{10}\right)^{1 / 2}$. For a $C O_{2}$ molecule $\hbar \omega_{32} \simeq \hbar \omega_{21} \simeq 700 \mathrm{~cm}^{-1}$ and under normal conditions $n_{20} / n_{10} \simeq 10 \%$. Using data on Raman cross section $\left(r_{13} \simeq 10^{-24} \mathrm{~cm}^{3}, \lambda=488 \mathrm{~nm}\right.$ [5]) one can estimate that a $10-$ femtosecond pulse provides inversion between vibrational levels $02^{\circ} 0$ and $01^{\circ} 0$ if its power exeeds $10^{12} \mathrm{~W} / \mathrm{cm}^{2}$. This value can be easily satisfied by modern femtosecond technique. 


\section{Dissociation of Molecules by Femtosecond Pulses}

The possibility of effective excitation of high vibrational levels makes topical the problem of transformation, for example, dissociation of molecules under the action of ultrashort laser pulse. Usually, collisionless excitation and dissociation of molecules by resonant radiation are investigated on the basis of the chain of equations for amplitudes of population probabilities of vibrational levels. This analisis is very complicated because of a large amount of levels which should be under consideration. In our case the condition $\tau_{p} \omega_{n m} \ll 1$ means that the result of interaction is not sensitive to the real vibrational structure of molecule spectrum and makes it convinient to investigate directly the equations for wave functions of vibrational Hamiltonian regarding it as a function of the distance between nuclei. The statement of the problem is quite similar to that solved by A. Migdal on excitation and ionization of atom after its nucleus received an instant impact [6, page 178]. For simplicity we consider two-atom molecule and assume the pulse to be so short that during its action the nuclei acquire some velocity but their positions relative to electrons have no time to change. If at the initial instant of time the molecule is at the ground state then just after the pulse action it remains at the same vibrational level and its wave function is related to the initial one as

$$
\varphi_{0}(Q, t=\infty)=\varphi_{0}(Q, t=-\infty) e^{-i \frac{P Q}{h}},
$$

where $\mathcal{P}=-\frac{1}{4}\left(\frac{\partial \kappa_{0}}{\partial Q}\right) \int_{-\infty}^{\infty}|E(t)|^{2} d t$ is the impulse transmitted to molecule under the action of ultrashort $\delta$-pulse; $\kappa_{0}$ is polarizability of the molecule, $Q$ is vibrational coordinate.

The quantum state described by the wave function $\varphi_{0}(Q, \infty)$ is not already a stationary state of the molecule after receiving an instant impulse $\mathcal{P}$. The transition of the system to the stationary state is accompanied by excitation of the upper vibrational levels and the probability to find molecule at the level $\mathrm{v}$ is determined by the coefficient of expansion of $\varphi_{0}(Q, \infty)$ into series in terms of the stationary wave functions of the vibrational Hamiltonian:

$$
w_{o v}=\left|\int_{-\infty}^{\infty} \varphi_{v}^{*}(Q) e^{-i \frac{P Q}{h}} \varphi_{o}(Q) d Q\right|^{2} .
$$

It is evident that the higher the pulse energy and consequently the transmitted momentum $\mathcal{P}$, the more is the difference between the quantum state of the molecule just after the pulse action and the stationary state and the higher are the levels to be involved into transition of the molecule to its stationary state.

Let us display it in framework of the model of anharmonic Morse oscillator, i.e. a particle of mass $\mathrm{M}$ which is moving in a field wth potential energy $U(R)=D\left(e^{-2 \alpha R}-2 e^{-\alpha R}\right)[6]$. The spectrum of positive energy of this particle is continuous and corresponds to a dissociated molecule. In the negative energy region there is a finite series of vibrational levels which is convergent to the dissociation energy D: $E_{v+1}-E_{v}=\hbar \omega_{0}-v \cdot \hbar \triangle \omega_{0}$, where $\omega_{0}=\left(E_{1}-E_{0}\right) / \hbar=\alpha(\sqrt{2 M D}-\alpha \hbar) / M$ is the fundamental frequancy of the oscillator and $\triangle \omega_{0}=\alpha^{2} \hbar / M$ is the anharmonism of the molecule. The wave functions of Morse oscillator are of the form $\varphi_{v}(Q) \sim e^{-\xi / 2} \xi^{s} F(-v, 2 s+1, \xi)$, where $\xi=\frac{2 \sqrt{2 M D}}{\alpha \hbar} e^{-\alpha Q}, s+v=s_{0}=\frac{\omega_{0}}{\Delta \omega_{0}}+\frac{1}{2} \simeq N$ and $N$ is a total number of the oscillator levels.

The problem of excitation of anharmonic oscillator levels by an ultrashort radiation $\delta$-pulse has an exact solution. The probability of finding the molecule at the level $v$ is 


$$
w_{o v}=\frac{\beta \sinh (\pi \beta)}{\pi \Gamma\left(2 s_{0}\right) v !} \cdot \frac{|\Gamma(i \beta+v)|^{2}\left|\Gamma\left(i \beta+2 s_{0}-v\right)\right|^{2}}{\Gamma\left(2 s_{0}-v\right)},
$$

or, neglecting the difference between $s_{0}$ and $\mathrm{N},-$ has the form

$$
w_{o v}=\frac{\pi \beta}{(2 N-1) ! \sinh (\pi \beta)} \cdot \frac{\prod_{n=1}^{2 N-1}\left[\beta^{2}+(n-v)^{2}\right]}{v !(2 N-v-1) !},
$$

where $\beta=\mathcal{P}(\alpha \hbar)^{-1} \ln ^{-1}\left[\frac{2 \sqrt{2 M D}}{\alpha \hbar}\right] \sim \int_{-\infty}^{\infty}|E|^{2} d t$.

For weak ultrashort pulses $(\beta \rightarrow 0)$ the probability of exciting vibrational levels is small and proportional to the square of the pulse energy: $1-w_{o 0}=\beta^{2} \psi^{\prime}(2 N)$.

With elevation of the pulse energy the population of high vibrational levels increases and, therefore, the dissociation of the molecule becomes more probable. In the limit of superpower ultrashort pulses $(\beta \gg 2 N)$ the probability of dissociation is exponentially close to unity:

$$
w_{D}=1-\sum_{v=0}^{N-1} w_{o v} \simeq 1-2 \pi\left[\frac{2^{N-1}}{(2 N-1) !}\right]^{2} \beta^{4 N-1} e^{-\pi \beta} .
$$

Thus, under the action of femtosecond laser pulse molecules can be effectively excited on high vibrational levels, and moreover, when the pulse energy exceeds the threshold

$$
\beta_{v}^{2}=\frac{(2 N-v)[2 v(N-v-1)-1]}{2(N-v)}
$$

the inversion takes place between levels $v$ and $v-1$.

In conclusion let us present some estimations. For the $\mathrm{CO}$ molecule which is active in Raman scattering we shall estimate the energy of the laser pulse which provides inversion between the lowest levels $(v=1) \rightarrow(v=0)$. Using data on Raman cross section $\left(\partial \kappa_{0} / \partial Q \simeq 10^{-16} \mathrm{~cm}^{2}, \lambda=\right.$ $488 \mathrm{~nm}[5])$ and spectroscopic parameters $\left(D=83777 \mathrm{~cm}^{-1}, \alpha=2.3904 \AA^{-1}\right)$ [7] we can estimate the pulse energy required for the inversion to be of the order of $0.1 \mathrm{~J} / \mathrm{cm}^{2}$.

The last estimation is connected with the energy of the ultrashort pulse which is necessary for an effective $\left(w_{D} \sim 1\right)$ dissociation of a two-atom molecule. As an example we consider the molecule of $J_{2}$ whose dissociation energy is relatively small $(D \simeq 1.5 \mathrm{eV})$ and is of the same order of magnitude as the spectral width of a femtosecond pulse. From the condition $\beta \geq 2 N$ we can obtain that the pulse energy required for dissociation exceeds $1 \mathrm{~J} / \mathrm{cm}^{2}$. This requirment is much stronger than the one for the inversion on the lowest levels but it can be satisfied by the modern femtosecond technique.

\section{Acknowledgments}

This research is supported in part by the Russian Basic Research Foundation under Grant No. 9302-14271 and in part by Wissenschaftskolleg in Berlin, Schweizerische National-VersicherungsGesellschaft, Alusuisse-Lonza Holding AG and Mr. Helmut Hartmann (Augsburg). We would like to thank Professor M.H. Rubin and Professor Y.H. Shih for their kind invitation to participate in the Workshop. One of the authors (V.I.) is grateful to the American Physical Society for travel support. 


\section{References}

[1] S.A. Akhmanov, V.A. Vysloukh and A.S. Chirkin, Optics of Femtosecond Laser Pulses. (Nauka, Moscow, 1988, in Russian).

[2] Y.X. Yan, E.B. Gamble and K.A. Nelson, J. Chem. Phys.83, 5391 (1985).

[3] E.M. Belenov, A.V. Nazarkin and I.P.Prokopovich, JETP Lett. (Russia) 55, 223 (1992).

[4] E.M. Belenov and A.V. Nazarkin, JETP Lett. (Russia) 53, 188 (1991).

[5] W.R. Fenner, H.A. Hyatt et al, J. Opt. Soc. Am. 63, 73 (1973).

[6] L.D. Landau and E.M. Lifshitz, Quantum Mechanics, (Nauka, Moscow, 1963, in Russian).

[7] I.I. Tugov, Proceedings of Lebedev Physics Institute.146, 17 (Nauka, Moscow, 1984, in Russian). 
SECTION 8

EPR PROBLEM, BELL'S INEQUALITIES

AND

MULTIPHOTON INTERFEROMETRY 


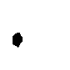

